\title{
Resistance to fluoroquinolones and second-line injectable drugs: impact on multidrug-resistant TB outcomes
}

\author{
Dennis Falzon', Neel Gandhi², Giovanni B. Migliori', Giovanni Sotgiu', \\ Helen S. Cox ${ }^{5}$, Timothy H. Holtz ${ }^{6}$, Maria-Graciela Hollm-Delgado ${ }^{7}$, \\ Salmaan Keshavjee ${ }^{8}$, Kathryn DeRiemer ${ }^{9}$, Rosella Centis $^{3}$, Lia D’Ambrosioº, \\ Christoph G. Lange ${ }^{10}$, Melissa Bauer ${ }^{7,11}$ and Dick Menzies ${ }^{7}$ on behalf of the \\ Collaborative Group for Meta-Analysis of Individual Patient Data in MDR-TB ${ }^{12}$
}

Affiliations: 'Stop TB Dept, World Health Organization, Geneva, Switzerland. ${ }^{2}$ Divisions of General Internal Medicine, Infectious Diseases and Epidemiology, Albert Einstein College of Medicine, New York, NY, ${ }^{8}$ Dept of Global Health and Social Medicine, Harvard Medical School, Boston, MA, and ${ }^{9}$ School of Medicine, University of California Davis, Davis, CA, USA. ${ }^{3}$ World Health Organization Collaborating Centre for Tuberculosis and Lung Diseases, Fondazione S. Maugeri, Care and Research Institute, Tradate, and ${ }^{4}$ Dept of Biomedical Sciences, University of Sassari, Sassari, Italy. ${ }^{5}$ Médecins Sans Frontières, Cape Town, South Africa. ${ }^{6}$ US Centers for Disease Control and Prevention, HIV/STD Research Program, Bangkok, Thailand. ${ }^{10}$ Clinical Infectious Diseases, Tuberculosis Center Borstel, Borstel, Germany. ${ }^{11}$ Dept of Epidemiology, Biostatistics, and Occupational Health, McGill University, Montreal, QC, and ${ }^{7}$ Montreal Chest Institute, McGill University, Montreal, QC, Canada. ${ }^{12} \mathrm{~A}$ full list of the Collaborative Group for Meta-Analysis of Individual Patient Data in MDR-TB members and their affiliations can be found in the Acknowledgements.

Correspondence: D. Menzies, Montréal Chest Institute, 3650 St Urbain St., Montréal, PQ, H2X 2P4, Canada. E-mail: Dick.MenziesaMcGill.ca

ABSTRACT A meta-analysis for response to treatment was undertaken using individual data of multidrug-resistant tuberculosis (MDR-TB) (resistance to isoniazid and rifampicin) patients from 26 centres. The analysis assessed the impact of additional resistance to fluoroquinolones and/or second-line injectable drugs on treatment outcome.

Compared with treatment failure, relapse and death, treatment success was higher in MDR-TB patients infected with strains without additional resistance $(n=4763 ; 64 \%, 95 \%$ CI $57-72 \%)$ or with resistance to second-line injectable drugs only $(n=1130 ; 56 \%, 95 \%$ CI $45-66 \%)$, than in those having resistance to fluoroquinolones alone $(n=426 ; 48 \%, 95 \%$ CI 36-60\%) or to fluoroquinolones plus second-line injectable drugs (extensively drug resistant (XDR)-TB) $(n=405 ; 40 \%, 95 \%$ CI 27-53\%). In XDR-TB patients, treatment success was highest if at least six drugs were used in the intensive phase (adjusted OR 4.9, 95\% CI 1.4-16.6; reference fewer than three drugs) and four in the continuation phase (OR 6.1, 95\% CI 1.4-26.3). The odds of success in XDR-TB patients was maximised when the intensive phase reached 6.6-9.0 months duration and the total duration of treatment 20.1-25.0 months.

In XDR-TB patients, regimens containing more drugs than those recommended in MDR-TB but given for a similar duration were associated with the highest odds of success.

All data were from observational studies and methodologies varied between centres, therefore, the bias may be substantial. Better quality evidence is needed to optimise regimens.

@ERSpublications

Resistance to fluoroquinolones and second-line injectable drugs have additive adverse impacts on MDR-TB outcomes http://ow.ly/kMDN8

For editorial comments see page 5.

This article has supplementary material available from erj.ersjournals.com

Received: Aug 272012 | Accepted after revision: Oct 052012 | First published online: Oct 252012

Conflict of interest: None declared. 


\section{Introduction}

The emergence of drug resistance among tuberculosis (TB) strains was first reported $>60$ years ago, soon after the introduction of the first antibiotics to treat TB [1-3]. Since then, broader patterns of drug resistance have been described worldwide, with the highest levels of resistance among TB patients being recorded in recent years [4]. In Belarus and other countries of the former Soviet Union, more than onequarter of treatment-naïve TB patients, and well over half of those who were previously treated, are now infected with strains resistant to both rifampicin and isoniazid (multidrug-resistant Mycobacterium tuberculosis (MDR-TB)) [5]. In 2010, there were an estimated 12 million prevalent TB cases globally, of which $\sim 650000$ were infected with MDR-TB strains. China and India are each estimated to have $>60000$ MDR-TB cases emerging annually from among the pulmonary TB patients that these countries notify [6]. Surveillance data from a number of settings indicate that, on average, 9.4\% (95\% CI 7.4-11.6\%) of MDRTB strains have additional resistance to both fluoroquinolones and second-line injectable drugs, i.e. extensively drug resistant (XDR)-TB [7]. The first reported outbreak of XDR-TB, which occurred in a high HIV-prevalence setting, was characterised by very high mortality [8]. Subsequent reports have confirmed that treatment outcomes for XDR-TB are generally worse than MDR-TB [9]. There is less information about the influence of individual resistance to fluoroquinolones and to second-line injectable drugs on prognosis in MDR-TB patients [10].

Treatment of MDR-TB is difficult. Current regimens, when compared to those used to treat drugsusceptible TB, are less effective but more costly, toxic and lengthy [11, 12]. Because there are no published randomised trials on the treatment of MDR-TB patients, the evidence supporting current recommendations is of low quality and based largely on observational studies [13]. This leads to considerable controversy regarding optimal treatment. There is even less evidence regarding treatment of patients with more advanced patterns of resistance, such as XDR-TB. As a result, the current World Health Organization (WHO) treatment recommendations for XDR-TB patients are based on expert opinion alone [11].

We conducted an individual patient data meta-analysis to explore the effect of patient characteristics, regimen composition and duration on treatment outcomes for MDR-TB patients grouped according to whether their infecting strains had additional resistance to either fluoroquinolones or second-line injectable drugs, or both (XDR-TB).

\section{Methods}

\section{Data collection}

The collection and analysis of the individual patient data was conducted to address specific questions developed by an expert guideline development group convened by the WHO to revise recommendations for treatment of drug-resistant TB [13]. The study was approved by the ethics review board committees of the Montréal Chest Institute and McGill University Health Centre (Montréal, Canada) and the local ethics review boards of participating centres, when necessary. The study was determined to be research not involving identifiable human subjects by the USA Centers for Disease Control and Prevention, because anonymised data originally collected for a different purpose were used.

The studies included in the individual patient data meta-analysis were identified from original studies published in three recent systematic reviews of MDR-TB treatment outcomes in MDR-TB patients [14-16]. These reviews searched the EMBASE and MEDLINE databases, the Cochrane Library and the ISI Web of Science, and included studies published after 1970 that reported original data with at least one treatment outcome that conformed with agreed definitions [17] for patients with bacteriologically confirmed MDRTB. All studies identified were from observational studies of patient groups; none were randomised trials. Most patients were treated with individualised regimens in specialised referral centres.

The additional inclusion criteria for this meta-analysis were that the study authors could be contacted; that they were willing to share their data; and that the cohort included $\geqslant 25 \mathrm{MDR}-\mathrm{TB}$ patients. Anonymised

Support statement: Funding for this study was provided in part by the Stop TB Department of the World Health Organization, through a grant from USAID. Funding for data gathering at participating centres was provided as follows: in the State of California from the Centers for Disease Control and Prevention Cooperative Agreement Funds; in Mexico (Veracruz) from the Mexican Secretariat of Health, the National Institutes of Health of the United States (A135969 and K01TW000001), the Wellcome Trust (176W009), the Howard Hughes Medical Institute (55000632) and the Mexican Council of Science and Technology (SEP 2004-C01-47499, FOSSIS 2005-2 (14475), (87332)); in South Africa from the South African Medical Research Council funding. M. Bauer and D. Menzies were supported by salary awards from the Fonds de Recherche en Santé de Québec; L. Shah was supported by the Canadian Institutes of Health Research (Canada Graduate Scholarship); N. Gandhi is the recipient of a Doris Duke Charitable Foundation Clinical Scientist Development Award; and G.B. Migliori and R. Centis were funded by the European Community's Seventh Framework Programme (FP7/2007-2013) under grant agreement FP7-223681. 
information provided included patient demographics (age and sex), clinical features (site of disease, pretreatment sputum smear results for acid-fast bacilli and culture, chest radiography, HIV infection, use of antiretroviral therapy (ART)), drug susceptibility test (DST) results (initial DST to all first- and second-line drugs used), treatment factors (drugs and duration for initial and continuous phases of treatment, and surgical resection) and treatment outcomes, including adverse events. Individual patients were excluded from the datasets if they had only extrapulmonary $\mathrm{TB}$ or were missing information on drug regimens received or on treatment outcome. We included only patients for whom DST results for at least one fluoroquinolone and one second-line injectable drug were available. Most centres tested for susceptibility to either amikacin or kanamycin; this analysis grouped resistance to these two aminoglycosides into one variable. In this study, amikacin, kanamycin and capreomycin, but not streptomycin, were considered second-line injectable drugs. The term macrolide refers to azithromycin, clarithromycin or roxithromycin. Later-generation fluoroquinolones refer to gatifloxacin, levofloxacin, moxifloxacin and sparfloxacin. Lowdose levofloxacin refers to a daily administration of $<750 \mathrm{mg}$. The drugs belonging to group 4 and group 5 used in patients included in this study are listed in online supplementary table S1 (data from [18]).

\section{Data analysis}

The methodology used for conducting the individual patient data meta-analysis was based on criteria established by the Cochrane collaboration [19], and is described in greater detail elsewhere [20]. We considered three elements of drug-exposure in our analysis: 1) individual drugs administered; 2) number of likely effective drugs used; and 3) duration of treatment regimen. A drug was considered as likely to be effective if DST results showed the strain to be susceptible. If a medication was reported as having been used at any time during treatment, then the patient was considered to have been exposed to the particular drug. The intervals used to analyse the duration of treatment (intensive phase and total) provided for a sizeable number of cases to be present in each of the subgroups.

We first estimated pooled proportions of cases with different drug resistance patterns using an across-centre binomial random effects meta-analysis (PROC NLMIXED in SAS version 9.2; SAS Institute, Cary, NC, USA). For the individual patient data meta-analysis we used random effects multivariable logistic regression (random intercept and random slope) with penalised quasi-likelihood in order to evaluate the impact of drug-exposure on treatment outcomes (using PROC GLIMMIX in SAS) [21-23]. Estimates were adjusted for five covariates: age, sex, HIV infection, extent of disease (a composite covariate scored by merging sputum-smear positivity and cavities on chest radiography) and previous history of TB treatment (which was a three-level variable: no previous TB treatment, previous TB treatment with first-line drugs and previous MDR-TB treatment with second-line drugs). Missing values were imputed for the five covariates used in multivariable analyses. For imputation we used the mean from the other members of the same cohort to which the individual belonged if more than half the cohort members had values for that variable, or the mean value from all individuals analysed. Adjusted odds ratios and their confidence intervals were used to report the associations between patient characteristics and outcomes in the different patient groups.

Treatment success was defined as cure or treatment completion [17] and was compared with 1) treatment failure, relapse or death for the analysis of individual drugs and number of drugs; and 2) failure or relapse for the analysis of duration of treatment. Patients who died or defaulted were not considered in the analysis on treatment duration because a number of studies recorded the actual rather than the planned length of treatment and, consequently, the duration was shortened by death or default.

\section{Results}

Study centres and patient characteristics

Individual data from MDR-TB patients in 31 centres were available for the analysis [24-55] (online supplementary table S2). Five centres did not have information about DST results for fluoroquinolones and/or second-line injectable drugs. In total, 6724 MDR-TB cases from the other 26 centres were included in the analysis. Patients were placed on treatment in the various cohorts between 1980 and 2009.22 centres reported at least one case of MDR-TB plus resistance to at least one second-line injectable drug only (MDRTB+INJr), 18 reported cases with MDR-TB plus fluoroquinolone resistance only (MDR-TB+FQr) and 17 centres had XDR-TB cases. The size of the cohorts in each centre ranged from one to $1786 \mathrm{MDR}-\mathrm{TB}$ cases. Overall, 4763 (71\%) patients had MDR-TB but were susceptible to both fluoroquinolones and second-line injectable drugs (MDR-TB only), 1130 (17\%) had MDR-TB+INJr, 426 (6\%) had MDR-TB+FQr and 405 $(6 \%)$ had XDR-TB.

The 6724 MDR-TB subjects had a mean \pm SD age of $39.5 \pm 13.5$ years, $69 \%$ were male, $70 \%$ had been treated previously for TB (60\% with first-line and 10\% with second-line drugs) and 11\% were HIV-infected (table 1). The age and sex profile was comparable between the patient groups. HIV infection was less frequent in MDR-TB+FQr (1.7\%) and MDR-TB+INJr (5.1\%) than in MDR-TB only patients (14\%). Fewer 
TABLE 1 Characteristics of multidrug-resistant tuberculosis (MDR-TB) patients with different resistance patterns of Mycobacterium tuberculosis

\begin{tabular}{|c|c|c|c|c|c|}
\hline & MDR-TB only & MDR-TB+INJr & MDR-TB+FQr & XDR-TB & $\begin{array}{c}\text { Total MDR-TB } \\
\text { cases }\end{array}$ \\
\hline Studies $\mathrm{n}$ & 26 & 22 & 18 & 17 & 26 \\
\hline \multicolumn{6}{|l|}{ Demographic characteristics } \\
\hline Age years & $39.2 \pm 13.5$ & $39.9 \pm 13.3$ & $41.6 \pm 14.3$ & $40.6 \pm 13.8$ & $39.5 \pm 13.5$ \\
\hline Male & $\overline{68}$ & $\overline{74}$ & $\overline{68}$ & $\overline{67}$ & $\overline{69}$ \\
\hline Pulmonary TB only & 97 & 97 & 96 & 97 & 97 \\
\hline Sputum-smear positive & 73 & 73 & 79 & 79 & 74 \\
\hline Cavities on chest radiography & 65 & 66 & 60 & 77 & 66 \\
\hline Extensive disease\# & 72 & 71 & 78 & 78 & 73 \\
\hline \multicolumn{6}{|l|}{ Previous TB treatment } \\
\hline None & 20 & 24 & 19 & 16 & 30 \\
\hline
\end{tabular}

Data are presented as mean \pm SD or $\%$, unless otherwise stated. Values shown were computed using simple pooling across all studies. Percentages were calculated on the number of patients in each group with information available. MDR-TB: multidrug-resistant TB (resistance to at least isoniazid and rifampicin); MDR-TB only: MDR-TB, but susceptible to fluoroquinolones, amikacin/kanamycin and capreomycin (at least one secondline injectable drug tested); MDR-TB+INJr: MDR-TB plus resistance to amikacin/kanamycin and/or capreomycin, but susceptible to fluoroquinolones; MDR-TB+FQr: MDR-TB plus resistance to any fluoroquinolone, but susceptible to amikacin/kanamycin and/or capreomycin (at least one second-line injectable drug tested); XDR-TB: extensively drug-resistant tuberculosis (MDR-TB plus resistance to any fluoroquinolone and any second-line injectable drug (amikacin/kanamycin and/or capreomycin)). \#: Extensive disease was defined as sputum-smear positive, or cavities on chest radiography if information about sputum-smear was missing. ": Previous tuberculosis (TB) treatment was defined as treatment with any TB drug for $\geqslant=1$ month. Previous treatment could be with first-line drugs or with $\geqslant 2$ second-line drugs for MDR. In some patients, information was only available that they had been treated previously for TB, but not whether this was with first- or second-line drugs.

than $10 \mathrm{HIV}$ infected patients received ART in total. XDR-TB cases were more likely to have cavities on chest radiography and to have been treated with second-line drugs than the other MDR-TB patients.

\section{Resistance patterns}

The majority of centres tested for susceptibility to a single fluoroquinolone, mostly ofloxacin, and very few for later-generation fluoroquinolones. Over 3000 patients had resistance to streptomycin, representing $61 \%$ of all those tested (table 2). Prevalence of streptomycin resistance was highest among patients with resistance to second-line injectable drugs (i.e. XDR-TB or MDR-TB+INJr). Resistance to both a second-line aminoglycoside (amikacin and/or kanamycin) and capreomycin occurred in 13\% of all patients, 30\% of XDR-TB and 33\% of MDR-TB+INJr. More than $90 \%$ of XDR-TB patients had strains resistant to six or more anti-TB drugs.

\section{Outcomes}

Overall, $62 \%$ of patients were successfully treated, in $7 \%$ treatment failed or the patient relapsed, $9 \%$ died and $17 \%$ defaulted (table 3 ). XDR-TB cases had the lowest rates of treatment success and the highest rates of failure, relapse and death. After adjustment for patient clinical characteristics and clustering by centres, treatment success was significantly lower in all three MDR-TB patient groups with additional resistance (table 4). Treatment success declined as drug resistance patterns advanced; the lowest odds of treatment success were seen with XDR-TB and were next lowest in patients with MDR-TB+FQr (fig. 1). Treatment success was also less likely in patients who were older, had more advanced disease, were HIV-infected or had a history of prior TB treatment, especially with second-line drugs.

\section{Treatment correlates with outcomes \\ Specific drugs and regimens}

Treatment regimens included ethambutol in $44 \%$ of all patients and pyrazinamide in $67 \%$ of all patients; over $85 \%$ received an injectable drug (in 14\% streptomycin only). Almost $90 \%$ of patients received a fluoroquinolone, but in only $5 \%$ was this a later-generation fluoroquinolone (online supplementary table S1). Fluoroquinolones were used less often if resistance to them was detected ( $73-76 \%$ versus $91-92 \%$ 
TABLE 2 Resistance to anti-tuberculosis drugs by multidrug-resistant tuberculosis (MDR-TB) patient group

\begin{tabular}{|c|c|c|c|c|c|}
\hline & MDR-TB only & MDR-TB + INJr & MDR-TB +FQr & XDR-TB & $\begin{array}{l}\text { Total MDR-TB } \\
\text { cases }\end{array}$ \\
\hline Cases $\mathbf{n}$ & 4763 & 1130 & 426 & 405 & 6724 \\
\hline \multicolumn{6}{|l|}{ First-line drugs } \\
\hline Pyrazinamide & $1052(41)$ & $556(70)$ & $234(58)$ & $211(69)$ & $2053(50)$ \\
\hline Ethambutol & $1524(51)$ & $845(76)$ & $296(74)$ & $295(81)$ & $2960(61)$ \\
\hline Streptomycin & $1534(51)$ & $960(86)$ & $226(53)$ & $291(78)$ & $3011(61)$ \\
\hline Amikacin/kanamycin & 0 & $1042(92)$ & 0 & $383(95)$ & $1425(21)$ \\
\hline Capreomycin & 0 & $399(42)$ & 0 & 104 (38) & $503(16)$ \\
\hline Amikacin/kanamycin and capreomycin & 0 & 311 (33) & 0 & $82(30)$ & $393(13)$ \\
\hline $\begin{array}{l}\text { Amikacin/kanamycin and capreomycin and } \\
\text { streptomycin }\end{array}$ & 0 & $295(31)$ & 0 & $68(25)$ & $363(12)$ \\
\hline TB drugs tested ${ }^{+}$ & $7.9 \pm 3.0$ & $10.0 \pm 1.3$ & $10.2 \pm 0.9$ & $9.6 \pm 1.7$ & $8.5 \pm 2.1$ \\
\hline \multicolumn{6}{|l|}{$\begin{array}{l}\text { Total number of TB drugs to which strain was } \\
\text { resistant }^{\S}\end{array}$} \\
\hline 2 & $2259(47)$ & 0 & 0 & 0 & 2259 (34) \\
\hline 3 & $947(20)$ & $15(1)$ & $19(4)$ & 0 & $981(15)$ \\
\hline 4 & $784(16)$ & $100(9)$ & $66(15)$ & $4(1)$ & $954(14)$ \\
\hline 5 & $513(11)$ & $331(29)$ & $101(24)$ & $32(8)$ & $977(15)$ \\
\hline 6 & $209(4)$ & $296(26)$ & $118(28)$ & $108(27)$ & $731(11)$ \\
\hline 7 & $42(1)$ & $221(20)$ & $89(21)$ & $105(26)$ & $457(7)$ \\
\hline 8 & $9(0.2)$ & $128(11)$ & $25(6)$ & $75(19)$ & $237(4)$ \\
\hline 9 & 0 & 37 (3) & $8(2)$ & $46(11)$ & $91(1)$ \\
\hline$\geqslant 10$ & 0 & $2(0.2)$ & 0 & 35 (9) & $37(0.3)$ \\
\hline
\end{tabular}

Data are presented as $\mathrm{n}, \mathrm{n}(\%)$ or mean \pm SD. Drug-susceptibility test results for Group 5 drugs were available from very few centres and were not analysed. $\mathrm{n}(\%)$ data are presented for the number of cases whose isolate was tested to that specific drug. All cases were tested for susceptibility to at least one fluoroquinolone (FQ) and one second-line injectable drug, but not all the other drugs. " : Most centres tested only for resistance to ofloxacin. Very few centres also tested for resistance to later-generation FQs (results of these tests are not shown). By definition, two patient groups were susceptible to $F Q$. ": Resistance to amikacin or kanamycin combined. Most centres tested for susceptibility to only one of these two drugs and considered them cross-resistant. ${ }^{+}$: Includes tests to isoniazid and rifampin, as well as to FQs and second-line injectable drugs (performed in all cases). ${ }^{\S}$ : In addition to isoniazid and rifampin, to which all patients were resistant, being MDR-TB. MDR-TB: multidrug-resistant tuberculosis (resistance to at least isoniazid and rifampicin); MDR-TB only: MDR-TB, but susceptible to FQs, amikacin/kanamycin and capreomycin lat least one second-line injectable drug tested); MDR-TB+INJr: MDR-TB plus resistance to amikacin/kanamycin and/or capreomycin, but susceptible to FQs; MDR-TB+FQr: MDR-TB plus resistance to any FQ, but susceptible to amikacin/kanamycin and/or capreomycin lat least one second-line injectable drug tested); XDR-TB: extensively drug-resistant tuberculosis (MDR-TB plus resistance to any FQ and any second-line injectable drug (amikacin/kanamycin and/or capreomycin)).

if susceptible). Capreomycin was given more often than amikacin/kanamycin to patients with MDRTB+INJr (56\% versus 22\%) and XDR-TB (40\% versus 33\%). Almost 95\% of patients in each subgroup received at least one group $4 \mathrm{drug}$, usually ethionamide or protionamide. Cycloserine or terizidone were given more often when MDR-TB patients had strains with additional resistance (84-89\% versus $58 \%$ ), as was $p$-aminosalicylic acid (46-64\% versus 35\%). Group 5 drugs were also used more frequently in the MDR-TB patients with additional resistance (36-44\%) than those without (18\%). $6 \%$ of all patients had adjunctive lung resection surgery; this was most frequent in patients with MDR-TB+FQr (online supplementary table S1).

Table 5 summarises the association of individual anti-TB drugs with treatment success compared to failure, relapse or death in the different MDR-TB patient groups. No drug was statistically significantly associated with treatment success among the MDR-TB+FQr or XDR-TB groups. In the MDR-TB+INJr group, amikacin or kanamycin (over streptomycin) and ethionamide or protionamide were significantly associated with treatment success. In the MDR-TB only patient group, the use of amikacin or kanamycin, 
TABLE 3 Treatment outcomes by multidrug-resistant tuberculosis (MDR-TB) patient group

\begin{tabular}{|c|c|c|c|c|c|}
\hline Pooled treatment outcomes ${ }^{\#}$ & MDR-TB only & MDR-TB +INJr & MDR-TB +FQr & XDR-TB & Total \\
\hline Subjects & 4763 & 1130 & 426 & 405 & 6724 \\
\hline Treatment success & $64(57-72)$ & $56(45-66)$ & $48(36-60)$ & $40(27-53)$ & $62(54-69)$ \\
\hline Died & $8(5-11)$ & $8(3-14)$ & $11(3-19)$ & $15(8-23)$ & $9(5-12)$ \\
\hline Defaulted & $18(12-24)$ & $16(7-24)$ & $12(1-23)$ & $16(8-24)$ & $17(11-22)$ \\
\hline
\end{tabular}

Data are presented as $\mathrm{n}$ or $\%$ (95\% CI). MDR-TB: multidrug-resistant TB (resistance to at least isoniazid and rifampicin); MDR-TB only: as MDR-TB, but susceptible to fluoroquinolones, amikacin/kanamycin and capreomycin (at least one second-line injectable drug tested); MDR-TB+INJr: MDRTB plus resistance to amikacin/kanamycin and/or capreomycin, but susceptible to fluoroquinolones; MDR-TB+FQr: MDR-TB plus resistance to any fluoroquinolone, but susceptible to amikacin/kanamycin and/or capreomycin lat least one second-line injectable drug tested); XDR-TB: extensively drug-resistant tuberculosis (MDR-TB plus resistance to any fluoroquinolone and any second-line injectable drug lamikacin/kanamycin and/or capreomycin)J. " : from study level meta-analysis; column percentages do not total $100 \%$. See the Methods section and [17] for treatment outcome definitions.

capreomycin, ofloxacin, ethionamide or protionamide and cycloserine were all associated with significantly higher odds of treatment success. Conversely, those patients in this group who received two group 5 drugs had a lower likelihood of treatment success than those receiving one group 5 drug, and so did those on a regimen without a fluoroquinolone or which contained only first-line drugs (online supplementary table S3). MDR-TB+INJr patients treated with a capreomycin-containing regimen fared worse than those who received kanamycin alone.

TABLE 4 Association of treatment success with patient characteristics and multidrug-resistant tuberculosis (MDR-TB) patient group

Cases

Adjusted odds of treatment success versus treatment failure/relapse/death"

\begin{tabular}{|c|c|c|}
\hline Male (versus female) ${ }^{\top}$ & 4653 & $1.0(0.9-1.1)$ \\
\hline Older age (per 10-year increment) & 6724 & $0.8(0.8-0.9)$ \\
\hline HIV infected (versus not HIV infected) & 615 & $0.3(0.2-0.4)$ \\
\hline Extensive disease (versus not extensive) & 4792 & $0.5(0.4-0.6)$ \\
\hline \multicolumn{3}{|l|}{ Previous TB treatment } \\
\hline None & 1275 & 1.0 (Reference \\
\hline First-line drugs only & 4410 & $0.6(0.5-0.8)$ \\
\hline First-line and second-line drugs & 618 & $0.2(0.15-0.3)$ \\
\hline \multicolumn{3}{|l|}{ MDR-TB patient group ${ }^{+}$} \\
\hline MDR-TB only & 4763 & 1.0 (Reference \\
\hline MDR+INJr & 1130 & $0.6(0.5-0.7)$ \\
\hline $\mathrm{MDR}+\mathrm{FQr}$ & 426 & $0.3(0.2-0.4)$ \\
\hline XDR-TB & 405 & $0.2(0.2-0.3)$ \\
\hline \multicolumn{3}{|c|}{ Pulmonary resection surgery performed (versus no } \\
\hline \multicolumn{3}{|c|}{ Experienced a serious adverse event (versus no serious } \\
\hline adverse event) ${ }^{+}$ & 1511 & $1.0(0.8-1.2)$ \\
\hline
\end{tabular}

Data are presented as $\mathrm{n}$ or adjusted OR (95\% Cl). TB: tuberculosis; MDR-TB: multidrug-resistant TB (resistance to at least isoniazid and rifampicin); MDR-TB only: MDR-TB, but susceptible to fluoroquinolones, amikacin/kanamycin and capreomycin lat least one second-line injectable drug tested); MDR-TB+INJr: MDR-TB plus resistance to amikacin/kanamycin and/or capreomycin, but susceptible to fluoroquinolones; MDRTB+FQr: MDR-TB plus resistance to any fluoroquinolone, but susceptible to amikacin/kanamycin and/or capreomycin lat least one second-line injectable drug tested); XDR-TB: extensively drug-resistant tuberculosis (MDR-TB plus resistance to any fluoroquinolone and any second-line injectable drug (amikacin/kanamycin and/or capreomycin)). \#: odds ratios of treatment success (cure and completion) versus treatment failure/ relapse/death adjusted for age, sex, HIV infection, previous TB treatment, previous MDR treatment (treatment for $>1$ month with two or more second-line drugs) and extent of disease. See Methods and [17] for treatment outcome definitions. ": estimate adjusted for all other covariates (characteristics) shown. ${ }^{+}$: each of these parameters estimated separately, and adjusted for age, sex, HIV, extent of disease and previous treatment with first- or second-line TB drugs. Statistical significance is represented by bold type. 


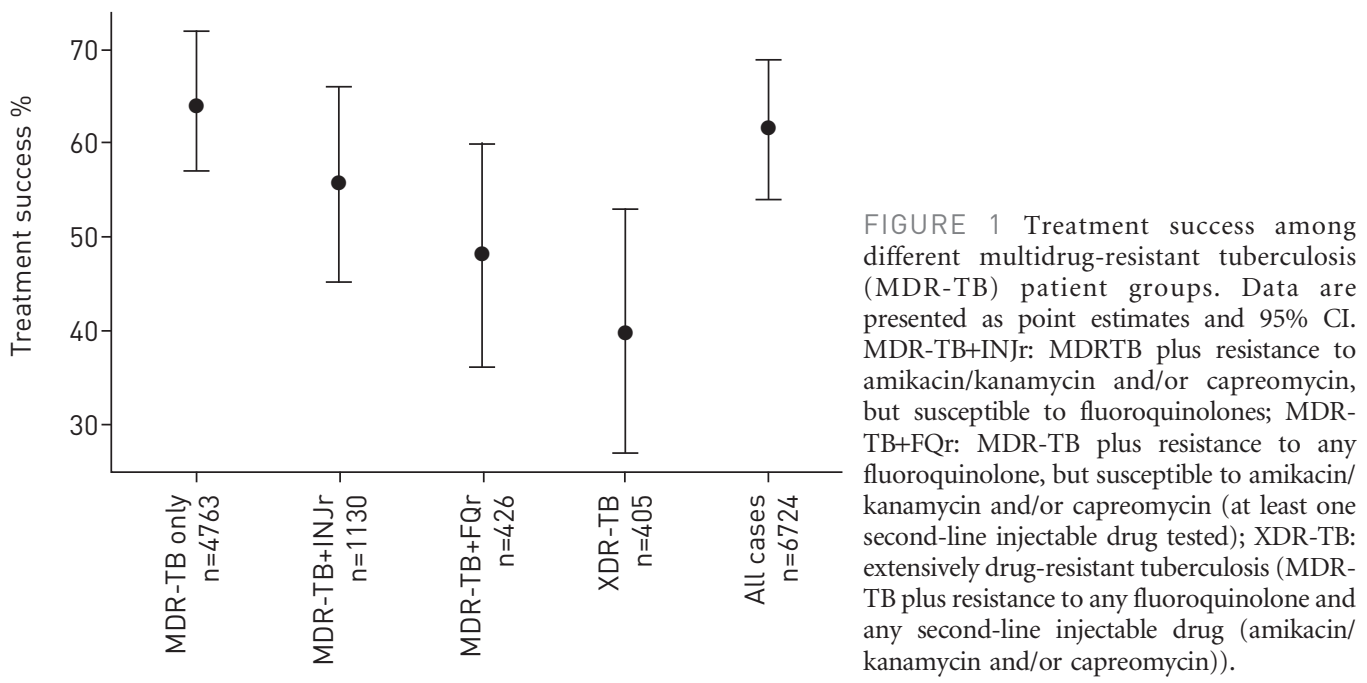

\section{Number of drugs and duration of treatment}

XDR-TB patients who in the intensive phase received six or more drugs, which were likely to be effective, and MDR-TB only patients who received four drugs, had a higher likelihood of treatment success than patients receiving fewer drugs (table 6). In the continuation phase, the use of four drugs for XDR-TB patients and three drugs for MDR-TB patients without fluoroquinolone-resistant strains were associated with the highest odds of treatment success.

Among all patients except those in the MDR-TB+FQr group, an intensive phase duration of 6.6-9.0 months was associated with the maximal odds of treatment success (statistically significant) compared with patients treated for shorter or longer durations (table 7). The odds of treatment success in the same three patient groups peaked when total duration of treatment was 20.1-25.0 months.

\section{Discussion}

We found a stepwise worsening of treatment outcomes in MDR-TB cases treated in multiple centres as the resistance pattern of infecting TB strains advanced from MDR without additional resistance, to added resistance to a second-line injectable drug, to resistance to a fluoroquinolone, and then to both (XDR-TB). This effect is attributable to the gradual loss of effectiveness of the two classes of medications that form the backbone of MDR-TB treatment. The negative impact on treatment success when isoniazid and rifampicin are lost to resistance was demonstrated several years ago [56]. Our findings complement those from published work on separate patient cohorts, which showed that resistance to fluoroquinolones or secondline injectable drugs in MDR-TB patients was associated with poorer prognosis $[57,58]$ and that outcomes for patients with XDR-TB are particularly unfavourable [8-10, 35, 40].

Current treatment guidelines for MDR-TB recommend the use of pyrazinamide along with at least four second-line TB medications likely to be effective given in vitro susceptibility results and prior treatment history [13]. A typical regimen can be created using a fluoroquinolone, a second-line aminoglycoside or capreomycin, ethionamide or protionamide and cycloserine or terizidone or $p$-aminosalicylic acid. With resistance to either fluoroquinolones or second-line injectable drugs, a regimen of four effective drugs is still possible without using any of the group 5 medications, most of which have uncertain activity against TB. However, with resistance to both of these drug classes, it becomes difficult to construct a tolerable regimen containing a sufficient number of effective drugs [11]. This difference in ability to create a robust treatment regimen may explain why treatment outcomes are so low in the XDR-TB group. The results of our metaanalysis indicate that in XDR-TB patients a regimen of a similar duration, but composed of more drugs than the regimen recommended for MDR-TB patients without additional resistance is more likely to achieve success [20].

In our study, we found that approximately one-third of patients tested for resistance to both the secondline aminoglycosides and capreomycin were resistant to drugs from both classes. This finding may suggest cross-resistance between these drug classes, which has been described, but is known not to be complete and is, therefore, less frequent [59]. However, it could also be explained by previous exposure 
TABLE 5 Association of treatment success with individual drugs used in treatment by multidrug-resistant tuberculosis (MDRTB) patient group

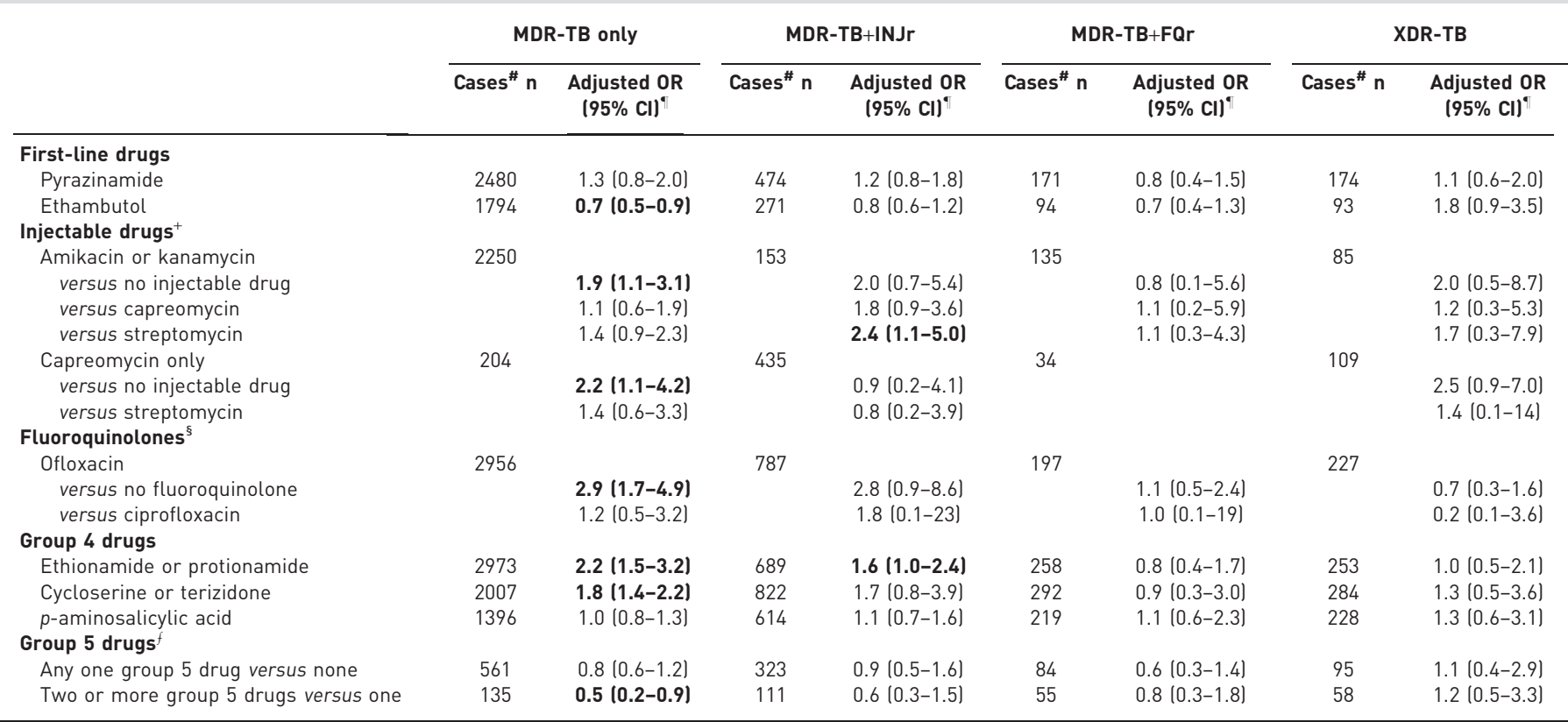

MDR-TB: multidrug-resistant TB (resistance to at least isoniazid and rifampicin); MDR-TB only: MDR-TB, but susceptible to fluoroquinolones, amikacin/kanamycin and capreomycin lat least one second-line injectable drug tested); MDR-TB+INJr: MDR-TB plus resistance to amikacin/kanamycin and/or capreomycin, but susceptible to fluoroquinolones; MDR-TB+FQr: MDR-TB plus resistance to any fluoroquinolone, but susceptible to amikacin/kanamycin and/or capreomycin lat least one second-line injectable drug tested); XDR-TB: extensively drug-resistant tuberculosis (MDR-TB plus resistance to any fluoroquinolone and any second-line injectable drug (amikacin/ kanamycin and/or capreomycin)). \#: number of cases that received the drug in question and were included in the specific analysis. ": odds ratios of treatment success (cure and completion) versus treatment failure/relapse/death adjusted for age, sex, HIV infection, previous TB treatment, previous MDR treatment (treatment for $>1$ month with two or more second-line drugs) and extent of disease. If there were $<50$ observations no estimate was derived. See Methods and [17] for treatment outcome definitions. ${ }^{+}$: patients receiving two or more injectable drugs were excluded from this analysis. ${ }^{\S}$ : patients receiving two or more fluoroquinolones were excluded from this analysis. Insufficient numbers of patients received later-generation fluoroquinolones (including gatifloxacin, levofloxacin, moxifloxacin and sparfloxacin) within the MDR-TB patient groups with additional resistance, so were not analysed. ${ }^{f}$ : insufficient numbers of patients received specific group 5 drugs within the MDR-TB patient groups with additional resistance, so outcomes by individual group 5 drugs were not analysed. Group 5 drugs included amoxicillin/clavulanate, macrolides (azithromycin, clarithromycin and roxithromycin), clofazimine, thiacetazone, imipenem, linezolid, high-dose isoniazid and thioridazine. Statistical significance is represented by bold type.

to both types of injectable drugs or to primary infection with a strain bearing this resistance pattern. Centres may use capreomycin empirically to treat cases with strains resistant to second-line aminoglycosides without the capacity to test for resistance to this drug. A number of patients received more than one type of injectable drug, but these were received sequentially, mostly because of DST results indicating resistance to the first injectable drug. Our findings suggest that capreomycin would probably not benefit such patients and could cause more harm than good, given the known toxicity of this agent. Patients on second-line medications often experience serious adverse events that require a change in therapy [60]. In our series an adverse event leading to a change in therapy occurred in $32 \%$ of cases overall.

Another important observation was that among patients with strains resistant to fluoroquinolones, secondline injectable drugs, or both, only one-quarter had been treated previously with second-line TB drugs. The rest were treated with first-line drugs or were never treated at all. This suggests that many of the MDR-TB cases with strains bearing additional resistance are due to primary infection with a resistant strain and, by inference, that the acquisition of drug resistance by a strain does not necessarily compromise its transmissibility [61]. Moreover, the propensity for XDR-TB strains to cause epidemics has been well recognised, particularly in settings with high HIV prevalence [8]. This finding reinforces the importance of having a comprehensive infection control component in all TB control programmes. Treatment of drugresistant $\mathrm{TB}$ patients with adequate regimens should also be instituted earlier, and scaled up globally to cover many more patients than the minority who are currently on appropriate treatment, particularly in high-burden settings $[6,62,63]$. In 2010, only $16 \%$ of MDR-TB cases estimated to occur among TB patients notified worldwide were reported to have been started on treatment. Moreover, the early use of ART in HIV-infected patients with MDR-TB is very important [13]. 
TABLE 6 Association of treatment success with the number of effective drugs used in the intensive and continuation phases of treatment by the multidrug-resistant tuberculosis (MDR-TB) patient group

\begin{tabular}{|c|c|c|c|c|c|c|c|c|}
\hline $\begin{array}{l}\text { Number of } \\
\text { drugs }\end{array}$ & \multicolumn{2}{|c|}{ MDR-TB only } & \multicolumn{2}{|c|}{ MDR-TB+INJr } & \multicolumn{2}{|c|}{ MDR-TB+FQr } & \multicolumn{2}{|c|}{ XDR-TB } \\
\hline \multicolumn{9}{|l|}{$\begin{array}{c}\text { Intensive } \\
\text { phase }\end{array}$} \\
\hline$\leqslant 2$ & 45 & 1.0 (reference) & 29 & 1.0 (reference) & 10 & 1.0 (reference) & 24 & 1.0 (reference) \\
\hline 5 & 296 & $1.7(0.8-3.8)$ & 137 & $1.2(0.4-3.4)$ & 35 & $1.4(0.3-6.4)$ & 36 & $1.8(0.5-6.6)$ \\
\hline$\geqslant 6$ & 380 & $1.0(0.5-1.8)$ & 120 & $1.3(0.5-3.3)$ & 27 & $1.1(0.4-2.9)$ & 20 & $4.9(1.4-16.6)$ \\
\hline \multicolumn{9}{|l|}{$\begin{array}{l}\text { Continuation } \\
\text { phase }^{+}\end{array}$} \\
\hline$\leqslant 2$ & 77 & 1.0 (reference) & 46 & 1.0 (reference) & 35 & 1.0 (reference) & 27 & 1.0 (reference) \\
\hline 3 & 133 & $5.9(3.1-11.0)$ & 33 & $12.2(3.4-44)$ & 27 & $2.5(0.8-7.4)$ & 32 & $3.3(1.3-8.5)$ \\
\hline
\end{tabular}

MDR-TB: multidrug-resistant TB (resistance to at least isoniazid and rifampicin); MDR-TB only: MDR-TB, but susceptible to fluoroquinolones, amikacin/kanamycin and capreomycin (at least one second-line injectable drug tested); MDR-TB+INJr: MDR-TB plus resistance to amikacin/ kanamycin and/or capreomycin, but susceptible to fluoroquinolones; MDR-TB+FQr: MDR-TB plus resistance to any fluoroquinolone, but susceptible to amikacin/kanamycin and/or capreomycin (at least one second-line injectable drug tested); XDR-TB: extensively drug-resistant tuberculosis (MDR-TB plus resistance to any fluoroquinolone and any second-line injectable drug lamikacin/kanamycin and/or capreomycin)). \#: odds ratios of treatment success (cure and completion) versus treatment failure/relapse/death adjusted for age, sex, HIV infection, previous TB treatment, previous MDR treatment (treatment for $>1$ month with two or more second-line drugs) and extent of disease. See Methods and [17] for treatment outcome definitions. ": the initial part of a course of treatment during which an injectable drug is given. ${ }^{+}$: the period immediately following the initial phase when no injectable drug is given. Only 18 studies provided information regarding drug susceptibility testing and the number of drugs in the intensive phase, while only 15 of these described the number of drugs in the continuation phase. Statistical significance is represented by bold type.

This study represents the largest known individual patient data meta-analysis for outcomes of MDR-TB cases with strains harbouring additional resistance. Patients were treated in multiple settings (online supplementary table S2), located in many countries and in all WHO regions, thus enhancing the generalisability of the findings. Detailed data, which were standardised as much as possible, were available for all cases. Differences in treatment regimens often reflected differences in treating physicians' opinions and past experiences. Hence this dataset included substantial variation in the approach to treatment, independent of differences in patient characteristics. We had the opportunity to examine how treatment correlates with outcomes, which would not be possible with single-centre reports.

Nevertheless, this study does suffer from a number of important limitations. While attempts were made to standardise the variables, residual heterogeneity in prior treatment for TB, diagnostic methods, additional drug resistance, drug quality, treatment regimens, drug dosages, frequency of administration and use of thoracic surgery complicate the pooling of observations. DST results to ethambutol, pyrazinamide and the group 4 drugs are known to be less accurate and reproducible than those for the drugs that define XDR-TB. As none of the studies were randomised controlled trials, substantial bias and confounding are expected and the quality of evidence would be considered low [64]. Patients with more advanced disease, or infected with strains having broader resistance and with a considerable previous treatment history may have been more likely to receive longer treatment with more drugs, since most of them received individualised regimens. Our findings that use of any group 5 drugs, or two group 5 drugs, were associated with worse treatment outcomes may reflect such bias, which cannot be adjusted for adequately in multivariable regression. Many of the patients with MDR-TB and fluoroquinolone resistance received early-generation fluoroquinolones, to which they were almost certainly resistant. Strains that develop resistance to early-generation fluoroquinolones may still show susceptibility to later-generation agents and DSTs to these agents should be performed, where possible [65]. The sparse use of later-generation fluoroquinolones may explain why no significant association was detected between their use and successful treatment outcome. Finally, most datasets lacked information on the timing of smear or culture conversion, which is considered useful in guiding the work of clinicians [11]. 
TABLE 7 Association between the duration of treatment and treatment success by multidrug-resistant tuberculosis (MDR-TB) patient group

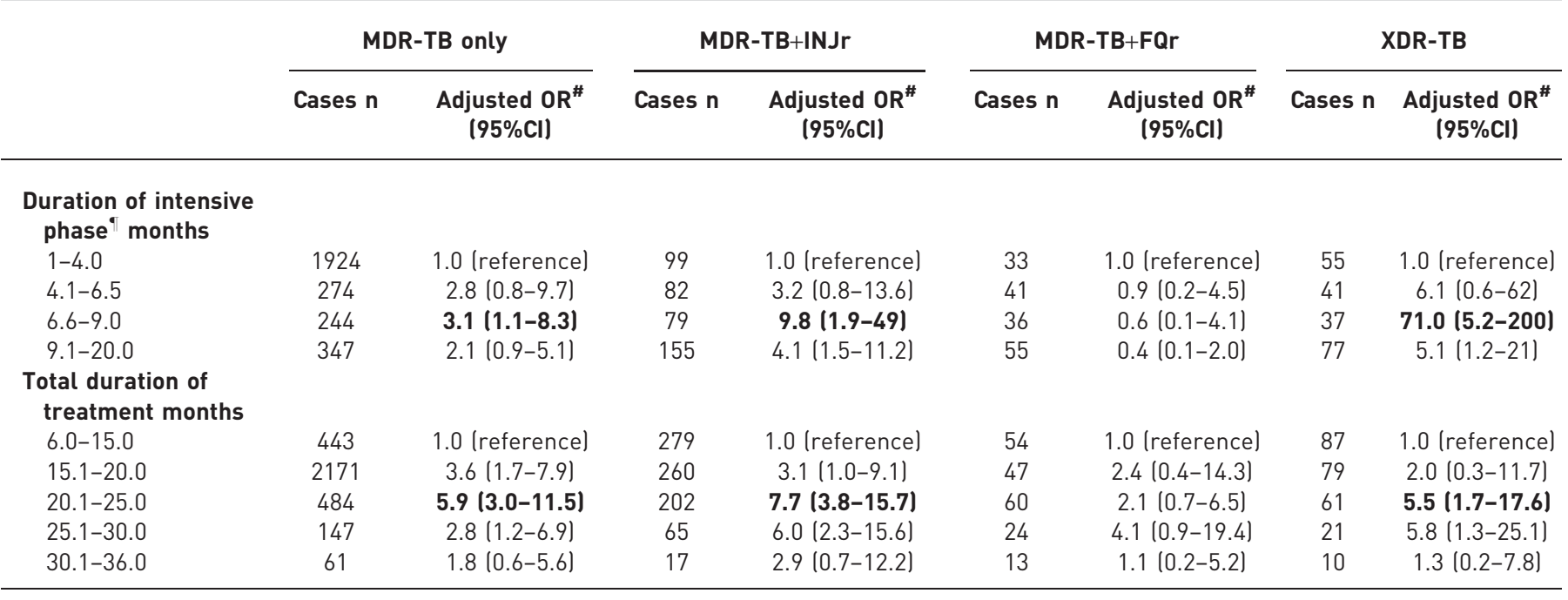

MDR-TB: multidrug-resistant TB (resistance to at least isoniazid and rifampicin); MDR-TB only: MDR-TB, but susceptible to fluoroquinolones, amikacin/kanamycin and capreomycin (at least one second-line injectable drug tested); MDR-TB+INJr: MDR-TB plus resistance to amikacin/ kanamycin and/or capreomycin, but susceptible to fluoroquinolones; MDR-TB+FQr: MDR-TB plus resistance to any fluoroquinolone, but susceptible to amikacin/kanamycin and/or capreomycin lat least one second-line injectable drug tested); XDR-TB: extensively drug-resistant tuberculosis (MDR-TB plus resistance to any fluoroquinolone and any second-line injectable drug lamikacin/kanamycin and/or capreomycin)). \#: Odds ratios of treatment success versus treatment failure or relapse adjusted for age, sex, HIV infection, previous TB treatment, previous MDR treatment (treatment for $>1$ month with two or more second-line drugs) and extent of disease. See Methods and [17] for treatment outcome definitions. ": The initial part of a course of treatment during which an injectable drug is given.

\section{Conclusions}

This analysis adds evidence about the detrimental effect of escalating resistance on TB treatment outcomes. The findings regarding the number of drugs and duration of treatment should be of use to clinicians when treating patients with drug-resistant $\mathrm{TB}$, but need to be interpreted with caution given the limitations mentioned. Randomised controlled trials are needed to optimise treatment regimens, including ancillary measures such as surgery. The addition of second-line drugs from the existent armamentarium of TB medications will only make a very modest difference once fluoroquinolones and second-line injectable agents are no longer an option. Better access for TB patients in resource-constrained settings to laboratories which can perform DST reliably, in order to detect resistance promptly, is very important [66]. New drugs that can be delivered in effective regimens are urgently needed to improve the outcomes of patients with the forms of drug-resistance described in this study [67].

\section{Acknowledgements}

The members of the Collaborative Group for Meta-analysis of Individual Patient Data in MDR-TB are as follows: S.D. Ahuja (Long Island City, NY, USA), D. Ashkin (Lantana, FL, USA), M. Avendaño (Toronto, ON, Canada), R. Banerjee (Rochester, MN, USA), M. Bauer (Montreal, QC, Canada), M.C. Becerra (Boston, MA, USA), A. Benedetti (Montreal, QC, Canada), M. Burgos (Albuquerque, NM, USA), R. Centis (Tradate, Italy), E.D. Chan (Denver, CO, USA), C.Y. Chiang (Taipei City, Taiwan), F. Cobelens, H. Cox (Cape Town, South Africa), L. D’Ambrosio (Tradate, Italy), W.C.M. de Lange (Haren, the Netherlands), K. DeRiemer (Davis, CA, USA), D. Enarson (Paris, France), D. Falzon (Geneva, Switzerland), K.L. Flanagan (Fajara, the Gambia), J. Flood (Richmond, CA, USA), N. Gandhi (Bronx, NY, USA), M.L. Garcia-Garcia (Cuernavaca, Mexico), R.M. Granich (Geneva, Switzerland), M.G. Hollm-Delgado (Montreal, QC, Canada), T.H. Holtz (Atlanta, GA, USA), P. Hopewell, M.D. Iseman (Denver, CO, USA), L.G. Jarlsberg (San Francisco, CA, USA), S. Keshavjee (Boston, MA, USA), H.R. Kim (Seoul, South Korea), W.J. Koh (Seoul, South Korea), J.L. Lancaster (Pretoria, South Africa), C. Lange (Borstel, Germany), V. Leimane (Upeslejas, Latvia), C.C. Leung (Hong Kong, China), J. Li (New York, NY, USA), D. Menzies (Montreal, QC, Canada), G.B. Migliori (Tradate, Italy), C.D. Mitnick (Boston, MA, USA), M. Narita (Seattle, WA, USA), E. Nathanson, R. Odendaal, P. O'Riordan (London, UK), M. Pai (Montreal, QC, Canada), D. Palmero (Buenos Aires, Argentina), S.K. Park (Masan City, South Korea), G. Pasvol (London, UK), J.M. Pena (Madrid, Spain), C. Pérez-Guzmán (Aguascalientes, México), A. Ponce-de-Leon (Mexico City, Mexico), M.I.D. Quelapio (Makati, Philippines), H.T. Quy, V. Riekstina (Upeslejas, Latvia), J. Robert (Paris, France), S. Royce (San Francisco, CA, USA), M. Salim, H.S. Schaaf (Tygerberg, South Africa), K.J. Seung (Boston, MA, USA), L. Shah (Montreal, QC, Canada), K. Shean, T.S. Shim (Seoul, South Korea), S.S. Shin (Boston, MA, USA), Y. Shiraishi (Tokyo, Japan), J. Sifuentes-Osornio (Mexico City, Mexico), G. Sotgiu (Sassari, Italy), M.J. Strand (Denver, CO, USA), S.W. Sung, P. Tabarsi (Tehran, Iran), T.E. Tupasi (Makati, Philippines), M.H. Vargas (Mexico City, Mexico), R. van 
Altena (Groningen, the Netherlands), M.L. van der Walt (Pretoria, South Africa), T.S. van der Werf (Groningen, the Netherlands), P. Viiklepp (Tallinn, Estonia), J Westenhouse (Richmond, CA, USA), W.W. Yew (Hong Kong, China), J.J. Yim (Seoul, South Korea).

The authors also thank the following individuals for help in the following ways: data gathering in the Philippines by R. Guilatco, G. Balane, and M. Galipot; data gathering in Toronto: M. Haslah, and J. McNamee, facilitation of the study at CDC (USA) by P. Lobue; assistance in data management by D. Weissman, S. Atwood, T. Buu, E. Desmond, M. KatoMaeda, J. Kirsten, and G. Lin; secretarial and administrative assistance by R. Choe and S. Ramoutar; statistical and logistic help with South African data from P. Becker.

Disclaimers: Dennis Falzon, Reuben M. Granich and Eva Nathanson are staff members of the World Health Organization (WHO). The authors alone are responsible for the views expressed in this publication and they do not necessarily represent the decisions or policies of WHO. Timothy H. Holtz is a staff member of the US Centers for Disease Control and Prevention (CDC). The author alone is responsible for the views expressed in this publication and they do not necessarily represent the decisions or policies of CDC.

\section{References}

Crofton J, Mitchison DA. Streptomycin resistance in pulmonary tuberculosis. BMJ 1948; 2: 1009-1015.

Crofton J. The chemotherapy of tuberculosis. With special reference to patients whose bacilli are resistant to the standard drugs. Br Med Bull 1960; 16: 55-60.

3 Crofton J. Drug treatment of tuberculosis. II. Treatment of patients with tubercle bacilli resistant to standard chemotherapy. BMJ 1960; 2: 449-451.

4 World Health Organization. Towards universal access to diagnosis and treatment of multidrug-resistant and extensively drug-resistant tuberculosis by 2015. WHO progress report 2011. Geneva, WHO Press, 2011.

5 Skrahina A, Hurevich $\mathrm{H}$, Zalutskaya A, et al. Alarming levels of drug-resistant tuberculosis in Belarus: results of a survey in Minsk. Eur Respir J 2012; 39: 1425-1431.

World Health Organization. Global tuberculosis control: WHO report 2011. Geneva, WHO Press, 2011.

Zignol M, van Gemert W, Falzon D, et al. Surveillance of anti-tuberculosis drug resistance in the world: an updated analysis, 2007-2010. Bull World Health Organ 2012; 90: 111D-119D.

8 Gandhi NR, Moll A, Sturm AW, et al. Extensively drug-resistant tuberculosis as a cause of death in patients coinfected with tuberculosis and HIV in a rural area of South Africa. Lancet 2006; 368: 1575-1580.

9 Jacobson KR, Tierney DB, Jeon CY, et al. Treatment outcomes among patients with extensively drug-resistant tuberculosis: systematic review and meta-analysis. Clin Infect Dis 2010; 51: 6-14.

10 Leimane V, Dravniece G, Riekstina V, et al. Treatment outcome of multidrug/extensively drug-resistant tuberculosis in Latvia, 2000-2004. Eur Respir J 2010; 36: 584-593.

11 World Health Organization. Guidelines for the programmatic management of drug-resistant tuberculosis, emergency update 2008. Geneva, WHO Press, 2008.

12 World Health Organization. Multidrug and extensively drug-resistant TB (M/XDR-TB): 2010 global report on surveillance and response. Geneva, WHO Press, 2010.

13 Falzon D, Jaramillo E, Schünemann HJ, et al. WHO guidelines for the programmatic management of drug-resistant tuberculosis: 2011 update. Eur Respir J 2011; 38: 516-528.

14 Johnston JC, Shahidi NC, Sadatsafavi M, et al. Treatment outcomes of multidrug-resistant tuberculosis: a systematic review and meta-analysis. PLoS One 2009; 4: e6914.

15 Orenstein EW, Basu S, Shah NS, et al. Treatment outcomes among patients with multidrug-resistant tuberculosis: systematic review and meta-analysis. Lancet Infect Dis 2009; 9: 153-161.

16 Akcakır Y. Correlates of treatment outcomes of multidrug-resistant tuberculosis (MDR-TB): a systematic review and meta-analysis. MSc Thesis. McGill University: Montréal, Canada. 2010.

17 Laserson KF, Thorpe LE, Leimane V, et al. Speaking the same language: treatment outcome definitions for multidrug-resistant tuberculosis. Int J Tuberc Lung Dis 2005; 9: 640-645.

18 World Health Organization. Guidelines for the programmatic management of drug-resistant tuberculosis, 2011 update. Geneva, WHO Press, 2011.

19 Higgins JPT, Green S. Cochrane Handbook for Systematic Reviews of Interventions. Oxford, The Cochrane Collaboration, 2011. www.cochrane-handbook.org Date last accessed: April 30, 2011. Date last updated: March 2011.

20 Ahuja SD, Ashkin D, Avendano M, et al. Multidrug resistant pulmonary tuberculosis treatment regimens and patient outcomes: an individual patient data meta-analysis of 9,153 patients. PLoS Med 2012; 9: e1001300.

21 Thompson SG, Turner RM, Warn DE. Multilevel models for meta-analysis, and their application to absolute risk differences. Stat Methods Med Res 2001; 10: 375-392.

22 Turner RM, Omar RZ, Yang M, et al. A multilevel model framework for meta-analysis of clinical trials with binary outcomes. Stat Med 2000; 19: 3417-3432.

23 Higgins JPT, Thompson SG. Quantifying heterogeneity in a meta-analysis. Stat Med 2002; 21: 1539-1558.

24 Avendaño M, Goldstein R. Multidrug-resistant tuberculosis: long term follow-up of 40 non-HIV-infected patients. Can Respir J 2000; 7: 383-389.

25 Burgos M, Gonzalez LC, Paz EA, et al. Treatment of multidrug-resistant tuberculosis in San Francisco: an outpatient-based approach. Clin Infect Dis 2005; 40: 968-975.

26 Chan ED, Laurel V, Strand MJ, et al. Treatment and outcome analysis of 205 patients with multidrug-resistant tuberculosis. Am J Respir Crit Care Med 2004; 169: 1103-1109.

27 Chiang C-Y, Enarson DA, Yu M-C, et al. Outcome of pulmonary multidrug-resistant tuberculosis: a 6-yr follow-up study. Eur Respir J 2006; 28: 980-985.

28 Cox HS, Kalon S, Allamuratova S, et al. Multidrug-resistant tuberculosis treatment outcomes in Karakalpakstan, Uzbekistan: treatment complexity and XDR-TB among treatment failures. PLoS One 2007; 2: e1126.

29 DeRiemer K, García-García L, Bobadilla-del-Valle M, et al. Does DOTS work in populations with drug-resistant tuberculosis? Lancet 2005; 365: 1239-1245. 
30 Escudero E, Peña JM, Alvarez-Sala R, et al. Multidrug-resistant tuberculosis without HIV infection: success with individualised therapy. Int J Tuberc Lung Dis 2006; 10: 409-414.

31 Geerligs WA, Van Altena R, De Lange WCM, et al. Multidrug-resistant tuberculosis: long-term treatment outcome in the Netherlands. Int J Tuberc Lung Dis 2000; 4: 758-764.

32 Granich RM, Oh P, Lewis B, et al. Multidrug resistance among persons with tuberculosis in California, 1994-2003. JAMA 2005; 293: 2732-2739.

33 Holtz TH, Lancaster J, Laserson KF, et al. Risk factors associated with default from multidrug-resistant tuberculosis treatment, South Africa, 1999-2001. Int J Tuberc Lung Dis 2006; 10: 649-655.

34 Kim DH, Kim HJ, Park S-K, et al. Treatment outcomes and long-term survival in patients with extensively drugresistant tuberculosis. Am J Respir Crit Care Med 2008; 178: 1075-1082.

35 Kim H-R, Hwang SS, Kim HJ, et al. Impact of extensive drug resistance on treatment outcomes in non-HIVinfected patients with multidrug-resistant tuberculosis. Clin Infect Dis 2007; 45: 1290-1295.

36 Kwon YS, Kim YH, Suh GY, et al. Treatment outcomes for HIV-uninfected patients with multidrug-resistant and extensively drug-resistant tuberculosis. Clin Infect Dis 2008; 47: 496-502.

37 Leimane V, Riekstina V, Holtz TH, et al. Clinical outcome of individualised treatment of multidrug-resistant tuberculosis in Latvia: a retrospective cohort study. Lancet 2005; 365: 318-326.

38 Lockman S, Kruuner A, Binkin N, et al. Clinical outcomes of Estonian patients with primary multidrug-resistant versus drug-susceptible tuberculosis. Clin Infect Dis 2001; 32: 373-380.

39 Masjedi MR, Tabarsi P, Chitsaz E, et al. Outcome of treatment of MDR-TB patients with standardised regimens, Iran, 2002-2006. Int J Tuberc Lung Dis 2008; 12: 750-755.

40 Migliori GB, Besozzi G, Girardi E, et al. Clinical and operational value of the extensively drug-resistant tuberculosis definition. Eur Respir J 2007; 30: 623-626.

41 Mitnick C, Bayona J, Palacios E, et al. Community-based therapy for multidrug-resistant tuberculosis in Lima, Peru. N Engl J Med 2003; 348: 119-128.

42 Munsiff SS, Ahuja SD, Li J, et al. Public-private collaboration for multidrug-resistant tuberculosis control in New York City. Int J Tuberc Lung Dis 2006; 10: 639-648.

43 Narita M, Alonso P, Lauzardo M, et al. Treatment experience of multidrug-resistant tuberculosis in Florida, 19941997. Chest 2001; 120: 343-348.

44 O’Riordan P, Schwab U, Logan S, et al. Rapid molecular detection of rifampicin resistance facilitates early diagnosis and treatment of multi-drug resistant tuberculosis: case control study. PLoS One 2008; 3: e3173.

45 Palmero DJ, Ambroggi M, Brea A, et al. Treatment and follow-up of HIV-negative multidrug-resistant tuberculosis patients in an infectious diseases reference hospital, Buenos Aires, Argentina. Int J Tuberc Lung Dis 2004; 8: 778-784.

46 Park SK, Lee WC, Lee DH, et al. Self-administered, standardized regimens for multidrug-resistant tuberculosis in South Korea. Int J Tuberc Lung Dis 2004; 8: 361-368.

47 Pérez-Guzmán C, Vargas MH, Martínez-Rossier LA, et al. Results of a 12-month regimen for drug-resistant pulmonary tuberculosis. Int J Tuberc Lung Dis 2002; 6: 1102-1109.

48 Quy HT, Cobelens FGJ, Lan NTN, et al. Treatment outcomes by drug resistance and HIV status among tuberculosis patients in Ho Chi Minh City, Vietnam. Int J Tuberc Lung Dis 2006; 10: 45-51.

49 Schaaf HS, Shean K, Donald PR. Culture confirmed multidrug resistant tuberculosis: diagnostic delay, clinical features, and outcome. Arch Dis Child 2003; 88: 1106-1011.

50 Shin SS, Pasechnikov AD, Gelmanova IY, et al. Treatment outcomes in an integrated civilian and prison MDR-TB treatment program in Russia. Int J Tuberc Lung Dis 2006; 10: 402-408.

51 Shiraishi Y, Nakajima Y, Katsuragi N, et al. Resectional surgery combined with chemotherapy remains the treatment of choice for multidrug-resistant tuberculosis. J Thorac Cardiovasc Surg 2004; 128: 523-528.

52 Tupasi TE, Gupta R, Quelapio MID, et al. Feasibility and cost-effectiveness of treating multidrug-resistant tuberculosis: a cohort study in the Philippines. PLoS Med 2006; 3: e352.

53 Uffredi M-L, Truffot-Pernot C, Dautzenberg B, et al. An intervention programme for the management of multidrug-resistant tuberculosis in France. Int J Antimicrob Agents 2007; 29: 434-439.

54 Yew WW, Chan CK, Leung CC, et al. Comparative roles of levofloxacin and ofloxacin in the treatment of multidrug-resistant tuberculosis: preliminary results of a retrospective study from Hong Kong. Chest 2003; 124: 1476-1481.

55 Yew WW, Chan $\mathrm{CK}$, Chau $\mathrm{CH}$, et al. Outcomes of patients with multidrug-resistant pulmonary tuberculosis treated with ofloxacin/levofloxacin-containing regimens. Chest 2000; 117: 744-751.

56 Mitchison DA, Nunn AJ. Influence of initial drug resistance on the response to short-course chemotherapy of pulmonary tuberculosis. Am Rev Respir Dis 1986; 133: 423-430.

57 Kim DH, Kim HJ, Park S-K, et al. Treatment outcomes and survival based on drug resistance patterns in multidrug-resistant tuberculosis. Am J Respir Crit Care Med 2010; 182: 113-119.

58 Migliori GB, Lange C, Centis R, et al. Resistance to second-line injectables and treatment outcomes in multidrugresistant and extensively drug-resistant tuberculosis cases. Eur Respir J 2008; 31: 1155-1159.

59 Brossier F, Veziris N, Aubry A, et al. Detection by GenoType MTBDRsl test of complex mechanisms of resistance to second-line drugs and ethambutol in multidrug-resistant Mycobacterium tuberculosis complex isolates. J Clin Microbiol 2010; 48: 1683-1689.

60 Bloss E, Kuksa L, Holtz TH, et al. Adverse events related to multidrug-resistant tuberculosis treatment, Latvia, 2000-2004. Int J Tuberc Lung Dis 2010; 14: 275-281.

61 Borrell S, Gagneux S. Infectiousness, reproductive fitness and evolution of drug-resistant Mycobacterium tuberculosis. Int J Tuberc Lung Dis 2009; 13: 1456-1466.

62 World Health Organization. Resolution WHA62.15. Prevention and control of multidrug-resistant tuberculosis and extensively drug-resistant tuberculosis. In: Sixty-second World Health Assembly: Resolutions and Decisions, Annexes. Geneva, World Health Organization, 2009. Available from: apps.who.int/gb/ebwha/pdf_files/WHA62REC1/WHA62_REC1-en.pdf Date last accessed April 11, 2012.

63 Zhao Y, Xu S, Wang L, et al. National survey of drug-resistant tuberculosis in China. N Engl J Med 2012; 366: 2161-2170. 
64 Guyatt GH, Oxman AD, Vist GE, et al. GRADE: an emerging consensus on rating quality of evidence and strength of recommendations. BMJ 2008; 336: 924-926.

65 Sirgel FA, Warren RM, Streicher EM, et al. gyrA mutations and phenotypic susceptibility levels to ofloxacin and moxifloxacin in clinical isolates of Mycobacterium tuberculosis. J Antimicrob Chemother 2012; 67: 1088-1093.

66 World Health Organization. Global tuberculosis report 2012. Geneva, WHO Press, 2012.

67 Diacon AH, Dawson R, von Groote-Bidlingmaier F, et al. 14-day bactericidal activity of PA-824, bedaquiline, pyrazinamide, and moxifloxacin combinations: a randomised trial. Lancet 2012; 380: 986-993. 